\title{
Visual detection and perceptual independence: Assessing color and form
}

\author{
DALE J. COHEN \\ University of North Carolina, Wilmington, North Carolina
}

\begin{abstract}
Visual stimuli are multidimensional. One important perceptual problem is to determine how the dimensions are combined. One important aspect of dimensional combination is whether the dimensions are perceptually independent or perceptually correlated. A new task is presented-the visual detection task-that directly assesses the degree of perceptual correlation between any two dimensions. Two experiments were conducted that assess the degree of perceptual correlation between form and color during the early stages of perceptual analysis. The results show that form and color are not perceptually independent. In addition, the pattern of perceptual correlation found indicates that form and color are not processed independently. The pattern of results constrains all models of early vision. A model of early vision based on active signal modulation is proposed.
\end{abstract}

Visual stimuli are multidimensional. Some of the dimensions of visual stimuli are form, color, texture, motion status, and so on. Ashby and Townsend (1986) make the point that "a fundamentally important problem is to determine how these dimensions are combined in perceptual processing" (p. 154). One important aspect of dimensional combination is the degree to which the dimensions are perceived autonomously. That is, the dimensions can be either perceptually independent or perceptually correlated. The dimensions are perceptually independent if "the probability of simultaneously perceiving both components $A$ and $B$ [of a two-dimensional stimulus $A B$ ] is equal to the probability of perceiving component $A$ times the probability of perceiving component $B$ " (Ashby $\&$ Townsend, 1986, p. 154). The dimensions are perceptually correlated if the perception of one component interacts with the perception of the other component.

Ashby and Townsend's (1986) general recognition theory provides a theoretically precise and mathematically rigorous definition of perceptual independence. In this paper, I use the general recognition theory to assess whether the feature dimensions color and form are perceptually independent during the initial stages of perceptual analysis. I will first review the statistical definition of perceptual independence. I will then review the psychophysical evidence addressing this issue. Finally, I will present a new task, the visual detection task, that can directly and systematically assess perceptual independence as a function of a stimulus trait of interest.

The author would like to thank Julian Keith and Jennifer Sheehan for their helpful comments and support at various stages of this project. Correspondence should be addressed to D. J. Cohen, Department of Psychology, University of North Carolina, Wilmington, NC 28403-3297 (e-mail: cohen@sol.cms.uncwil.edu).

\section{PERCEPTUAL INDEPENDENCE}

Ashby and Townsend's (1986) general recognition theory is primarily formulated within the context of a signal detection model. Although they have generalized this theory to reaction times (RTs), the RT model makes more assumptions than does the signal detection model. Therefore, I will review only the signal detection model.

The signal detection model states that sensitivity for two feature dimensions, $A_{i}$ and $B_{j}$, fall in a two-dimensional space (Ashby \& Townsend, 1986; Green \& Swets, 1966; Kadlec \& Townsend, 1992; Macmillan \& Creelman, 1991). Figure 1 illustrates a model in which the distributions $A_{1} B_{1}, A_{1} B_{2}, A_{2} B_{1}, A_{2} B_{2}$ are bivariate normal distributions. The circles represent the distributions around the means. The distances between the means of the distributions are represented by double-sided arrows. The angle between the two dimensions is represented by theta $(\theta)$. The marginal distributions of the perceptual effects of each dimension $A_{i} B_{j}$ are represented on the bottom and right side of the figure.

Each axis of Figure 1 represents the perceptual values of the feature dimensions $A$ and $B$. Although theoretically each feature dimension may be composed of multiple dimensions (e.g., the feature dimension color is composed of intensity, hue, and saturation), the addition of the extra dimensions in the model is not necessary if one is only interested in whether any dimension of feature dimension $A$ interacts with any dimension of feature dimension $B$. Interactions between dimensions of feature dimension $A$ (or $B$ ) will not affect the conclusions (Ashby \& Townsend, 1986).

In the perceptual space described in Figure 1, perceptual independence of dimensions $A$ and $B$ holds if the two following criteria are validated: (1) the distributions for dimensions $A$ and $B$ are orthogonal (i.e., $\theta=90^{\circ}$ ), and (2) the variances of the distributions for one dimension are 


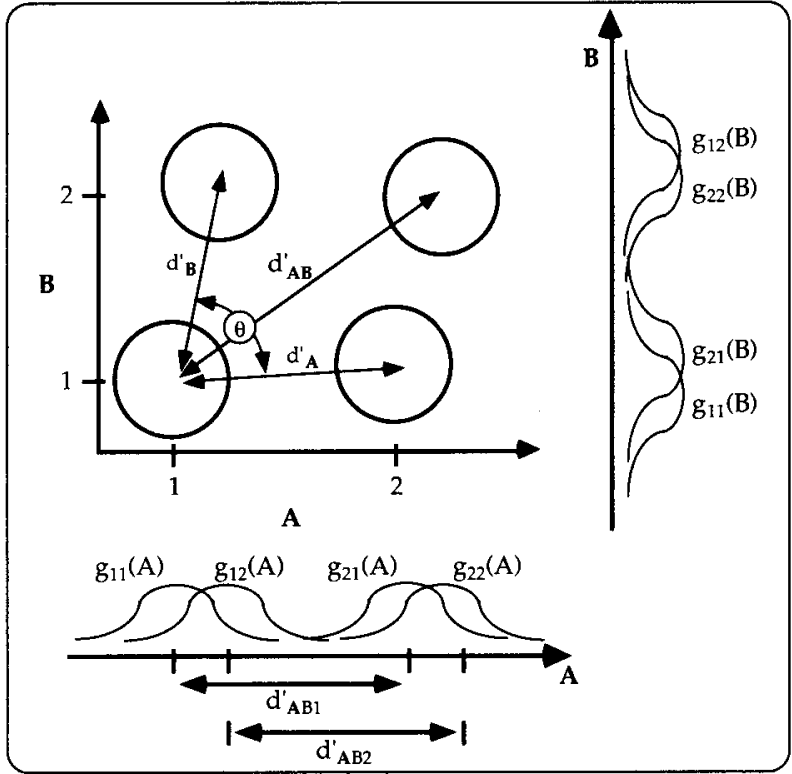

Figure 1. Two-dimensional space representing a subject's sensitivity to feature dimensions $A$ and $B$. Bottom and right represent the marginal densities and associated $d$ 's. From "Signal Detection Analysis of Dimensional Interactions," by $\mathrm{H}$. Kadlec \& J. T. Townsend, 1992, in F. G. Ashby (Ed.), Multidimensional Models of Perception and Cognition (p. 190), Hillsdale, NJ: Erlbaum. Copyright 1992 by Lawrence Erlbaum Associates. Reprinted with permission.

not influenced by the other dimension (i.e., all distributions are bivariate normal). Figure 2 displays examples of perceptual spaces in which each of these criteria holds or fails. If either one of these criteria fails, then perceptual independence of dimensions $A$ and $B$ fails.

The general recognition theory integrates many popular tests of perceptual independence, such as dimensional orthogonality, stimulus separability, performance parity, and feature sampling independence. Although any single test of perceptual independence cannot confirm perceptual independence, a combination of tests can. In addition, some single tests of perceptual independence can falsify perceptual independence. The experiments in this paper test the dimensional orthogonality of color and form. Therefore, I will only summarize the mathematical model for dimensional orthogonality.

\section{DIMENSIONAL ORTHOGONALITY}

The dimensional orthogonality test assesses the magnitude of $\theta$ in the perceptual space described in Figure 1. Because the dimensional orthogonality test does not assess the variances of the distributions, it alone cannot confirm perceptual independence. However, if dimensional orthogonality fails, perceptual independence of the dimensions is falsified.

Dimensional orthogonality is assessed by estimating the distances between the means of the distributions associated with dimensions $A_{i}$ and $B_{j}$. These distances can be estimated within the context of a signal detection model that quantifies the distances in terms of the $d^{\prime}$ measure. This can be accomplished by estimating subjects' ability to detect dimensions $A_{i}$ and $B_{j}$ using a standard signal detection procedure. For example, suppose one creates a factorial combination of two feature dimensions ( $A$ and $B$ ), each having two values ( 1 and 2 ). From the resulting stimulus set, $A_{1} B_{1}, A_{1} B_{2}, A_{2} B_{1}, A_{2} B_{2}$, a target is defined as any stimulus that has value 2 . The distance between the means of the perceptual distributions for $A_{1} B_{1}$ and $A_{1} B_{2}\left(d_{B}^{\prime}\right)$ can be estimated using a signal detection procedure that requires subjects to differentiate the two stimuli. Similar estimates can be made for the distance between $A_{1} B_{1}$ and $A_{2} B_{1}\left(d_{A}^{\prime}\right)$ and between $A_{1} B_{1}$ and $A_{2} B_{2}\left(d_{A B}^{\prime}\right)$.

Using the estimated distances between distributions (i.e., $d_{A}^{\prime}, d_{B}^{\prime}$, and $d_{A B}^{\prime}$ ), one can estimate the magnitude of $\theta$. In the two-dimensional space described above (Figure 1), the angle between the dimensions can be estimated using the Pythagorean relation (Ashby \& Townsend, 1986; Green \& Swets, 1966; Kadlec \& Townsend, 1992; Macmillan \& Creelman, 1991):

$$
\left(d_{A B}^{\prime}\right)^{2}=\left(d_{A}^{\prime}\right)^{2}+\left(d_{B}^{\prime}\right)^{2}+2 d_{A}^{\prime} d_{B}^{\prime} \cos \theta
$$

In such a model, $\cos \theta$ equals the perceptual correlation between dimensions $A$ and $B\left(\rho_{A B}\right)$. If the dimensions are independent, then $\rho_{A B}=0, \theta=90^{\circ}$, and the formula reduces to

$$
d_{A B}^{\prime}=\sqrt{\left(d_{A}^{\prime}\right)^{2}+\left(d_{B}^{\prime}\right)^{2}} .
$$

Therefore, the following criterion must be met if dimensions $A$ and $B$ are perceptually independent: The sensitivity of the system to a target that contains both signals is predicted by the square root of the sum of the squared sensitivity to each individual signal (Equation 1.1b).

If dimensional orthogonality fails, then perceptual independence is falsified. However, if dimensional orthogonality holds, perceptual independence is not con-

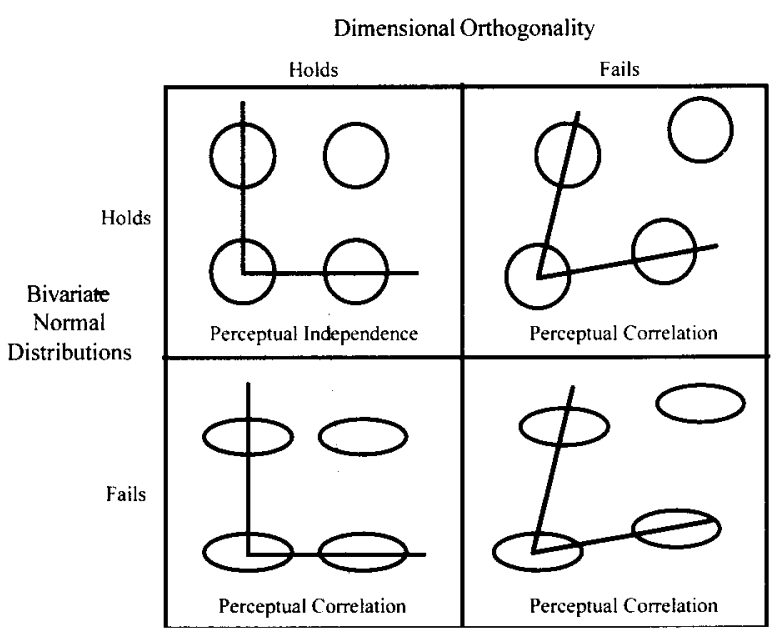

Figure 2. Examples of perceptual spaces in which dimensional orthogonality and bivariate normal distributions hold or fail. If either one of these criteria fails, perceptual independence of dimensions $A$ and $B$ fails. 
firmed, because the distributions' variances were not tested for normality. A separate test of the variances must be made. Below, I review the recent literature that addresses the question of perceptual independence between color and form.

\section{PSYCHOPHYSICAL EVIDENCE}

There have been no published reports that test color and form for dimensional orthogonality. However, there have been attempts to determine whether color and form are perceptually independent. It is important to note that, in the experiments below, color refers to the combination of hue, intensity, and saturation. That is, the colors were not isoluminant. Although some of these experiments have used accuracy as the dependent measure, most have used RT. The experiments that attempt to determine whether color and form are perceptually independent fall into two categories: (1) tests of sampling independence, and (2) tests of separability and integrality. The tests of separability and integrality can be divided into two subtests: (1) tests for inhibition between dimensions, and (2) tests of redundancy gain.

\section{Sampling Independence}

At issue with sampling independence is whether the identification of an object's color is independent of the identification of the object's form. For sampling independence to hold, the probability that a subject correctly specifies the color of an object should not be related to the probability that the subject correctly specifies the form of the object. An example of the failure of sampling independence is if a subject is more likely to correctly specify the color of an object when that subject correctly specifies the form of that object.

To test sampling independence of color and form, researchers have assessed subjects' accuracy when identifying the color and form of a briefly presented stimulus. For example, Nissen (1985) and Isenberg, Nissen, and Marchak (1990) presented subjects with an array of colored lines in different orientations, followed by both a mask and a position probe. The subjects' task was to identify both the color and the orientation of the probed stimulus. The results showed that, statistically, the subjects' identification of color and form was functionally independent. Recently, Monheit and Johnson (1994) disputed this conclusion. They increased the statistical power of Nissen's procedure and found a statistical dependence between the identification of color and form.

Closely related to sampling independence is the study of illusory conjunctions. Illusory conjunctions are the incorrect perceptual combination of feature dimensions when a target stimulus is briefly presented among distractor stimuli (A. Cohen \& Ivry, 1989; Ivry \& Prinzmetal, 1991; Treisman \& Schmidt, 1982). One may argue that if color and form information are sampled independently, one would predict the possibility of illusory conjunctions. However, the converse is not necessarily true: The failure of sampling independence does not predict the absence of illusory conjunctions. Butler, Mewhort, and Browse (1991) provide evidence that "illusory conjunctions do not reflect the system's architecture; they depend on the subject's encoding and selection strategy" (p. 98). That is, one can devise a system in which sampling independence fails and illusory conjunctions occur. Therefore, the existence of illusory conjunctions does not confirm sampling independence.

\section{Separability Versus Integrality}

At issue in the separability versus integrality debate is whether or not two dimensions, $A_{i}$ and $B_{j}$, can be separately attended to (Ashby \& Townsend, 1986). If the two dimensions can be attended to separately, they are considered separable and therefore provided some support for perceptual independence. Otherwise, the dimensions are integral, thus providing evidence against perceptual independence. As with the other tests of perceptual independence, tests of separability versus integrality cannot alone confirm perceptual independence. If dimensions are separable, then one would predict neither dimensional interference nor redundancy gains.

\section{Dimensional Interference}

To test dimensional interference, researchers have assessed the speed of detecting a target distinguished by a variation on feature dimension $A$, while manipulating whether feature dimension $B$ is heterogeneous or homogeneous. If the dimensions are separable, then the degree of heterogeneity of the irrelevant feature dimension should not affect detection of the target. For example, Pashler (1988) presented subjects with an array of $90 \mathrm{el}-$ ements that may or may not contain a target. The subjects' task was to identify whether a target was present in the display. The subjects' RTs were recorded. The elements varied on two feature dimensions (form and color), and each feature dimension had two feature values. The subjects were assigned a target feature value in one dimension (e.g., green) and were told to ignore the other dimension. On half of the trials, the irrelevant feature dimension was homogeneous. On the remaining trials, the irrelevant feature dimension was heterogeneous (i.e., the feature value of the irrelevant feature dimension was inverted on one element). The results revealed that, in the heterogeneous condition, the irrelevant feature dimension interfered with the detection of the target. This contradicts the prediction of separable dimensions. Interference between dimensions has been observed between form and color (Callaghan, 1989; Callaghan, Lasaga, \& Garner, 1986; Kubovy \& D. Cohen, 1991, 1992; Pashler, 1988) and between color and intensity (Callaghan, 1984).

\section{Redundancy Gains}

If separability between dimensions holds, then when two target values are presented, they should be detected independently. Any performance over that predicted by independence is considered a redundancy gain. For example, integrality between dimensions may predict that the two target values coactivate each other so as to pro- 
duce a signal stronger than that predicted by independent processing (synergy). For this reason, the term coactivation has been used to indicate redundancy gains.

To test the coactivation hypothesis, researchers have assessed the speed of detecting at least one target, while manipulating whether there are one or two targets present in the display. When two targets are present, either both targets are distinguished from the distractors by a single feature dimension (within-dimensions experiments) or each target is distinguished from the distractors by a different feature dimension (between-dimensions experiments). The subjects' RTs are recorded. All serial processing models (e.g., separability) predict that the fastest RT to a trial containing two targets should be no faster than the fastest RT to a trial containing a single target (Miller, 1982). For example, Mordkoff and Yantis (1993) presented subjects with a single element that may or may not contain a target value. The element varied on two feature dimensions (form and color), and each feature dimension had 2 feature values. The subjects were assigned a target feature value in each dimension (e.g., green or " $X$ "). The subjects were to identify whether at least one target value was present. The subjects' RT were recorded. The results showed that the subjects' fastest RT to a trial containing redundant target values (e.g., a green " $X$ ") was faster than the fastest trial containing a single target value. This contradicts the prediction of separable dimensions. Redundancy gains have only been revealed in betweendimensions experiments (for color/form, Mordkoff \& Yantis, 1993; for form/audition, Miller, 1982, 1991); no redundancy gain is found when the redundant target is in the same feature dimension (Duncan, 1980, 1985; Eriksen, Goettl, St. James, \& Fournier, 1989; Grice, Canham, \& Boroughs, 1984; Mordkoff \& Yantis, 1991; Mordkoff, Yantis, \& Egeth, 1990; van der Heijden, Maris, \& Neerincx, 1984).

\section{Summary of Psychophysical Findings}

Taken together, the recent psychophysical evidence suggests that color and form are not perceptually independent at the early stages of perceptual analysis. Unfortunately, while the RT independence analysis described above has furthered our understanding of this issue, it requires many processing assumptions that are controversial (see Ashby \& Townsend, 1986; Eriksen, 1988; Schwarz, 1989). In addition, RT analysis does not assess either (1) the precise processing time needed before perceptual correlation emerges or (2) the magnitude of that correlation. I describe below a new task, the visual detection task, that directly assesses the degree of perceptual correlation between two dimensions as a function of $X$, where $X$ is a stimulus trait of interest. This paradigm assesses both redundancy gains and inhibition in the same experiment. The estimated $d$ 's lie on a ratio measurement scale and allow one to assess the magnitude of $\rho_{A B}$ (i.e., the perceptual correlation between dimensions $A$ and $B$; see Dimensional Orthogonality section above).

\section{THE VISUAL DETECTION TASK}

The visual detection task is similar to the traditional visual search task in that it involves presenting a subject with a matrix of elements, which may or may not contain a target. The subjects' task is to judge whether a target is present. The difference between the two tasks lies in the composition of the matrix of elements and the description of the target.

In the visual detection task, there are two target values, one feature value from each feature dimension being tested for independence. A set of stimuli is constructed such that the stimuli are differentiated by two feature dimensions, $A_{i}$ and $B_{j}$, each having two feature values, $i=1,2$, and $j=1,2$. Within this set, one target feature value from each feature dimension is chosen, $A_{2}$ and $B_{2}$. Any stimulus that contains a target value is a target stimulus. Thus, there are three target stimuli, $A_{2} B_{1}, A_{1} B_{2}$, and $A_{2} B_{2}$, and one distractor, $A_{1} B_{1}$. So, if one is testing form (feature values, circle and square) and color (feature values, black and white) for independence, the target values may be black or square (Figure 3 ). A positive trial would contain any stimulus that is black or square (black circle, black square, or white square). The distractor must be a white circle.

Each trial can contain one or more of each target, or no target. So, there are at least five trial types: single target $A_{2} B_{1}$, single target $A_{1} B_{2}$, single target $A_{2} B_{2}$, dual targets $A_{2} B_{1}$ and $A_{1} B_{2}$, and no target $A_{1} B_{1}$ (Figure 4 shows these trial types in the context of the form/color example described above).

- There are two trial types that contain a single target that is differentiated from the distractors by the target feature value of one feature dimension:

$$
\begin{aligned}
& \text { (1) single target } A_{2} B_{1} \text {, and } \\
& \text { (2) single target } A_{1} B_{2} .
\end{aligned}
$$

In the form/color example, these trials would contain either a single target that has only the form target feature value (a white square) or a single target that has only the color target feature value (a black circle).

- There are two trial types that contain targets that are differentiated from the distractor by the target feature values in both feature dimensions:

$$
\text { (3) single target } A_{2} B_{2} \text {. }
$$

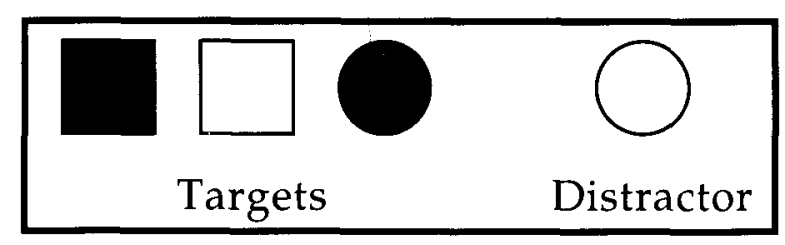

Figure 3. Example targets and distractor for the visual detection task. 


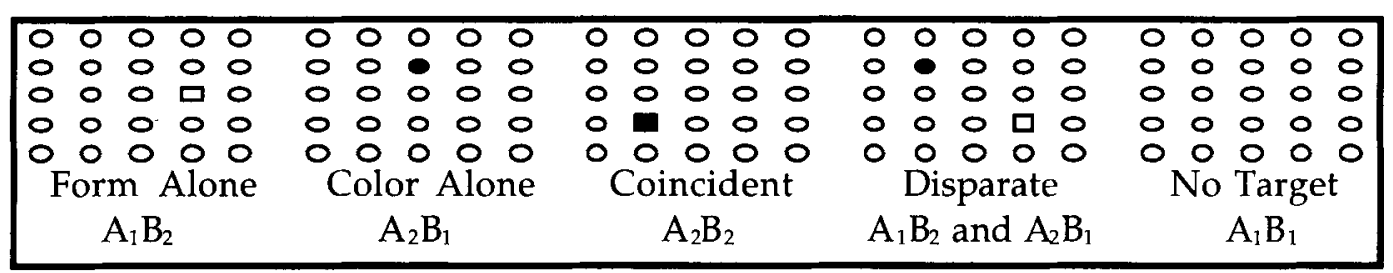

Figure 4. The five display types for the visual detection task.

The trial containing the single-target $A_{2} B_{2}$ is termed a coincident trial since the differentiating feature values are in coincident spatial locations. In the form/ color example, the single target would be a black square.

\section{(4) dual targets $A_{2} B_{1}$ and $A_{1} B_{2}$.}

The trials containing the dual targets $A_{2} B_{1}$ and $A_{1} B_{2}$ are termed disparate trials since the differentiating feature values are in different spatial locations. In the form/color example, the two targets would be a white square and a black circle.

- The no target trials (negative trials) contain no target features:

$$
\text { (5) only stimulus } A_{1} B_{1} \text {. }
$$

In the form/color example, the display would have only white circles.

Each trial contains a fixed number of stimuli and a fixed presentation time. The subjects' task is to judge whether at least one target feature value is present in the trial. So, the subjects give a positive response if they detect a stimulus that contains any target value (in the form/color example, black circle, black square, or white square). If no target is detected, then the subjects give a negative response.

On those trials where at least one target is present, the target is presented in a randomly chosen spatial position. This discourages the subjects from focusing their attention on a restricted portion of the display. Each positive trial type is presented with a probability of .125 , and the negative trials are presented with a probability of .5. Randomly presenting the trial types discourages subjects from relying on a trial-specific strategy.

\section{STATISTICAL ANALYSIS}

The data will be analyzed using a signal detection model. The analysis will consist of two tests that assess perceptual independence. First, the data will be analyzed to determine whether dimensional orthogonality holds (see Dimensional Orthogonality section above). Second, the data will be analyzed to determine whether spatial independence holds (described below).

To determine whether dimensional orthogonality holds, one must estimate the distances between the means of the distributions of the perceptual space described in Figure 1. A direct measure of the distance between the $A_{1} B_{1}$ distribution and the $A_{2} B_{1}$ and $A_{1} B_{2}$ distributions ( $d_{A}^{\prime}$ and $d_{B}^{\prime}$ ) can be derived from the single-target $A_{2} B_{1}$ and singletarget $A_{1} B_{2}$ trials. From these sensitivity measures, one can predict how well a subject should detect two signals if the signals are processed independently $\left(d_{\text {independence }}^{\prime}\right)$. This prediction can be made from Equation 1.1b. A direct measure of the distance between the $A_{1} B_{1}$ distribution and the $A_{2} B_{2}$ distribution ( $d_{A B}^{\prime}$ ) can be derived from the dual-target $A_{2} B_{2}$ trials. The visual detection task contains two dual-target trial types: coincident and disparate trials. The distance between the $A_{1} B_{1}$ distribution and each $A_{2} B_{2}$ trial type will be calculated separately because there is no a priori reason to assume that the perceptual spaces for these two trial types are identical. To assess whether perceptual independence holds, either one can solve Equation 1.1a for $\cos \theta$ for each perceptual space associated with the coincident and disparate stimuli or $d_{\text {independence }}^{\prime}$ can be compared with the direct measure of how well the visual system detects the two signals $\left(d_{A B}^{\prime}\right)$ derived from the coincident ( $d_{\text {coincident }}^{\prime}$ ) and disparate $\left(d_{\text {disparate }}^{\prime}\right)$ trials.

The test of spatial independence is derived from the notion that if two dimensions are perceptually independent, then no quality of one dimension should affect detection of the other dimension (Ashby \& Townsend, 1986). Therefore, if $A_{i}$ and $B_{j}$ are perceptually independent, then relative spatial location of the target feature values should be of no consequence, and the following criterion must be met:

$$
d_{A B}^{\prime}=d_{\text {disparate }}^{\prime}=d_{\text {coincident }}^{\prime} .
$$

These two tests alone cannot confirm perceptual independence. However, the failure to satisfy either dimensional orthogonality or spatial independence indicates that perceptual independence fails. The failure to satisfy both criteria provides strong evidence against perceptual independence.

\section{EXPERIMENT 1}

In Experiment 1, I used the visual detection task to test whether form and color are processed independently during the initial stage of perceptual analysis. To ensure that the initial, unlimited-capacity stage of perceptual analysis (often termed preattentive vision) is being assessed, I presented the stimuli for 33 and $83 \mathrm{msec}$. These presentation times preclude eye movements. The stimuli in this experiment are equivalent to those used in the studies cited above (both those claiming to support and those claim- 
ing to refute the perceptual independence between color and form). That is, the colors used were not isoluminant.

\section{Method}

Subjects. Seventy-two subjects from the General Psychology subject pool volunteered to participate. The data of 5 subjects were discarded because they were unable to perform the task.

Apparatus and Stimuli. All stimuli were presented on a 13-in. VGA color monitor with a $60-\mathrm{Hz}$ refresh rate controlled by an 80386 microcomputer using the DOS operating system. The resolution of the monitor was $640 \times 480$.

Each trial matrix consisted of a 5-square matrix of elements. The shape of each element was either square (28 pixels per side) or octagonal (the oblique lines were 3 pixels long, and the vertical and horizontal lines were 22 pixels long). The color of each element was either light blue (IBM palette No. 1) or dark blue (IBM palette No. 17). The matrix was centered on the monitor. The matrix subtended about $9.5^{\circ}$ visual angle, and each element subtended about $1^{\circ}$ visual angle. The feature values for each feature dimension were chosen on the basis of the subjects' performance on a pilot experiment. In the pilot experiment, I systematically adjusted the similarity between the two form feature values and the similarity between the two color feature values until most subjects could detect the form and color single-feature targets approximately equally well. The final feature values were used in the present experiment.

Target/distractor sets were counterbalanced between subjects. The subjects' target feature values did not change during the experiment. The position of the target element(s) was randomly chosen on each positive trial.

Each trial matrix was presented for either 33 or $83 \mathrm{msec}$. The trial matrix was followed by a mask that remained visible until the subjects made their judgments. The mask was a 5 -square matrix of red eight-pointed stars. The stars were the same size and in the same position as the matrix elements.

The subjects responded by pressing either the $\mathrm{D}$ or $\mathrm{K}$ key on the computer keyboard. The key indicating a positive response was randomly chosen for each subject.

Each subject participated in a single $40-\mathrm{min}$ session. The sessions consisted of 32 practice trials and 800 experimental trials. Negative trials were randomly presented with a probability of .5 . Each positive trial (single-target trials, $A_{1} B_{2}$, and $A_{2} B_{1}$; dual-target trials, $A_{1} B_{2}$ and $A_{2} B_{1}$, and $A_{2} B_{2}$ ) was randomly presented with a probability of 125 . The trial matrices were randomly presented for a stimulus onset asynchrony (SOA) of 33 or $83 \mathrm{msec}$ (with a probability of .5). Thus, there were 50 trials for each positive trial matrix $\times$ SOA, and 200 trials for each negative trial matrix $\times$ SOA.

Procedure. Each subject was tested individually in a small dark room. The subject was given both verbal and written instructions. The instructions indicated which target feature values the subject was to search for and indicated that the subject was to judge whether or not at least one target feature value was present in the display. The subject was shown self-timed examples of each trial type. When the subject indicated understanding of the task, the experiment began.

Each trial began with a 500-msec blank screen, followed by the trial matrix, followed by the mask. The mask remained visible until the subject responded. After the subject responded, feedback was visually presented (either CORRECT or INCORRECT was displayed on an otherwise blank screen) for $500 \mathrm{msec}$. The subject was allowed a self-timed break after every 200 trials.

\section{Results}

For each subject $\times$ trial type $\times$ SOA, a $d^{\prime}$ was calculated. In addition, for each subject $\times$ SOA, a $d_{\text {independence }}^{\prime}$ was calculated from the single-target $d^{\prime}$ s (using Equation 1.1b). For each subject $\times$ SOA, three $t$ values were calculated: $d_{\text {coincident }}^{\prime}-d_{\text {independence }}^{\prime} ; d_{\text {disparate }}^{\prime}-d_{\text {independence }}^{\prime}$; and $d_{\text {coincident }}^{\prime}-d_{\text {disparate }}^{\prime \prime}$. Since the data were not stable enough to provide meaningful results for individual subjects, a summed $z$ meta-analysis was performed. Median $d^{\prime} \mathrm{s}$ for SOA $\times$ trial type, as well as predicted independence, are presented in Table 1 . The important columns are those comparing the dual-target trials with independence.

Because a meta-analysis is not typically in the psychophysicist's repertoire of data analytic techniques, I will spend some time explaining the technique. In short, a meta-analysis is used to combine effect sizes of experiments to determine if an overall effect exists. Although the meta-analysis is traditionally used to concatenate effect sizes between similar experiments, it is also appropriate to use a meta-analysis within an experiment. In essence, the meta-analysis answers the question, "What is the probability that there would exist effect sizes of this magnitude in this direction for $N$ subjects?" To answer this question for the present experiment, the summed $z$ metaanalysis sums the $t$ values over subjects $\times$ SOA and then divides the result by the square root of the number of subjects (Rosenthal \& Rubin, 1986; Strube, 1985; Strube \& Miller, 1986). The summed $z$ analysis has been shown to compute results that are equivalent to other meta-analytic procedures (Strube \& Miller, 1986). The assumptions of the meta-analytic technique are (1) independent tests and (2) equivalent experimental designs. My data do not violate these assumptions: Each subject's data are independent of other subjects' data, and the experimental designs are identical. One advantage of using a meta-analysis over tests of mean differences is the added power it provides when individual subjects are tested many times (e.g., a large number of trials). A test of mean differences gains power only from the number of subjects. The meta-analysis analyzes the effect size for each subject and, thus, gains power from both the number of subjects and the number of trials per subject. By analyzing effect sizes, the metaanalysis emphasizes individual effects and not group effects. The disadvantage of the meta-analysis is that it does not estimate a group mean. Thus, a meta-analysis should not be used if quantification of the group mean is important. In the present experiment, a group mean is meaningless because each subject's sensitivity to each target is different. Thus, meta-analysis is preferable over a test of mean differences when the data show small but consistent effect sizes (because a test of mean differences may not have the power to confirm the effect), and an estimate of the group mean is unimportant. Finally, the analysis of effect sizes instead of mean differences has recently been proposed as one solution for the problems

Table 1

Median Predicted Independence and Median $d^{\prime}$ Scores for Each Positive Trial Matrix

\begin{tabular}{cccccc}
\hline $\begin{array}{c}\text { Presentation } \\
\text { Time }(\mathrm{msec})\end{array}$ & $\begin{array}{c}\text { Form } \\
\text { Alone }\end{array}$ & $\begin{array}{c}\text { Color } \\
\text { Alone }\end{array}$ & Disparate & $\begin{array}{c}\text { Predicted } \\
\left(\rho_{A B}=0\right)\end{array}$ & Coincident \\
\hline 33 & 0.39 & 0.45 & 0.93 & 0.99 & 1.03 \\
83 & 0.98 & 0.90 & 1.62 & 1.67 & 1.80 \\
\hline
\end{tabular}


associated with Null Hypothesis Statistical Testing (J. Cohen, 1994).

There were no significant differences between predicted independence and the two dual-target trials when the trial matrix was presented for $33 \mathrm{msec}$. When the trial matrix was presented for $83 \mathrm{msec}$, there was no significant difference between $d_{\text {disparate }}^{\prime}$ and predicted independence. However, for an SOA of $83 \mathrm{msec}$, the subjects were significantly more sensitive to the coincident trials than to both the disparate trials $[t(66)=2.06, p=.04]$ and predicted independence $[t(66)=2.86, p=.006]$.

\section{Discussion}

The results indicate that when a trial matrix is presented for an SOA of $33 \mathrm{msec}$, dimensional orthogonality and spatial independence hold. When the trial matrix is presented for an SOA of $83 \mathrm{msec}$, perceptual independence failed. At $83 \mathrm{msec}$, the subjects performed better on the coincident trials than on both the disparate trials and predicted independence. This is a violation of both dimensional orthogonality and spatial independence. If the subjects had searched for the target, one would predict that they should perform better on the disparate trials since there were target feature values in two spatial locations (and, thus, the probability of detection in a random serial self-terminating search or a search for simple heterogeneity is twice that of a trial that contains a target in a single spatial location). Thus, the subjects did not use focused attention to locate the targets. The implication of these results will be discussed in the General Discussion section.

Although there were no significant effects for an SOA of $33 \mathrm{msec}$, the subjects displayed a pattern of results consistent with the failure of perceptual independence. Given the quick presentation times, which result in both increased variability and small effect sizes, I ran a second experiment with more subjects to increase the power of the tests.

\section{EXPERIMENT 2}

Experiment 2 was conducted in order to expand and replicate the results of Experiment 1. In Experiment 2, more subjects were included in order to get a more powerful test of independence at $33 \mathrm{msec}$. In addition to the positive trial matrices used in Experiment 1, three new positive trial matrices were introduced. Two positive trial matrices contained two single-feature targets each $\left(A_{1} B_{2}\right.$ and $A_{1} B_{2} ; A_{2} B_{1}$ and $A_{2} B_{1}$ ), and one contained two dualfeature targets $\left(A_{2} B_{2}\right.$ and $\left.A_{2} B_{2}\right)$. The trial matrices that contained two single-feature targets were included to test within-dimension combination of information. Because the two single-feature targets are identical, they are (presumably) processed in the same system. Therefore, if relative spatial location is not important during the early stages of perceptual analysis, $\rho_{A B}$ should equal 1 , and subjects should be twice as sensitive to these targets than to an identical individual target (i.e., from Equation 1.1 a, if $\rho_{A B}=1$, then $\cos \theta=0$, and, therefore, Equation 1.1 a reduces to $d_{A B}^{\prime}=d_{A}^{\prime}+d_{B}^{\prime}$ ). The trial matrix that contained two dualfeature targets was included for the sake of consistency.

\section{Method}

Subjects. Ninety-eight subjects from the General Psychology subject pool volunteered to participate. The data of 8 subjects were discarded because they were unable to perform the task.

Apparatus and Stimuli. All stimuli were presented on a 13-in. VGA color monitor with a $60-\mathrm{Hz}$ refresh rate controlled by an 80486 microcomputer using the DOS operating system. The resolution of the monitor was $640 \times 480$.

The stimuli were identical to those in Experiment 1 with the exception that there were seven types of positive trials: the four positive trial types from Experiment l, (1) $A_{1} B_{2}$, (2) $A_{2} B_{1}$, (3) $A_{1} B_{2}$ and $A_{2} B_{1}$, and (4) $A_{2} B_{2}$; and three additional positive trials that contained redundant targets, (5) $A_{1} B_{2}$ and $A_{1} B_{2}$, (6) $A_{2} B_{1}$ and $A_{2} B_{1}$, and (7) $A_{2} B_{2}$ and $A_{2} B_{2}$. The additional positive trial matrices were included to test the effect of redundancy on within-module detection. The negative trials were presented with a probability of .5 , and each positive trial was presented with a probability of about .071 .

Target/distractor sets were counterbalanced between subjects. The subjects' target feature values did not change during the experiment. The position of the target element(s) was randomly chosen on each positive trial.

The subjects responded by pressing either the $\mathrm{D}$ or $\mathrm{K}$ key on the computer keyboard. The key indicating a positive response was randomly chosen for each subject.

Each subject participated in a single 40-min session. The sessions consisted of 56 practice trials and 980 experimental trials. Thus, there were 35 trials for each positive trial matrix $\times$ SOA, and 245 trials for each negative trial matrix $\times$ SOA.

Procedure. Between 1 and 4 subjects were run simultaneously, each in a separate, walled cubicle in a dark room. The subjects were given both verbal and written instructions. The instructions indicated which target feature values the subject was to search for and that the subject was to judge whether at least one target feature value was present in the display. The subject was shown self-timed examples of each trial type. When the subject indicated understanding of the task, the experiment began.

Each trial began with a 500-msec blank screen, followed by the trial matrix, followed by the mask. The mask remained visible until the subject responded. After the subject responded, feedback was visually presented (either CORRECT or INCORRECT was displayed on an otherwise blank screen) for $500 \mathrm{msec}$. The subject was allowed a self-timed break after every 200 trials.

\section{Results}

The data were analyzed using the same statistical procedure used in Experiment 1. Predicted independence for the trial matrices that contained two single feature target values was calculated using the following formula: $d_{A A}^{\prime}=2 d_{A}^{\prime}$ (from Equation 1.1 a, $\cos \theta=1$, since two identical target feature values are processed in the same system). Median $d$ 's for SOA $\times$ target trial type, as well as predicted performance, are presented in Tables 2, 3, and 4 . The important columns are those comparing the dual-target trials with predicted performance.

The subjects performed significantly worse than predicted when the trial matrix contained two elements with the color target feature value for both an SOA of $33 \mathrm{msec}$ $[t(89)=-10.05, p<.001]$ and an SOA of $83 \mathrm{msec}$ $[t(89)=-22.22, p<.001]$. The subjects performed significantly worse than predicted when the trial matrix con- 
Table 2

Median Predicted Performance and Median $d^{\prime}$ Scores for the Color Positive Trial Matrices

\begin{tabular}{cccc}
\hline $\begin{array}{c}\text { Presentation } \\
\text { Time }(\mathrm{msec})\end{array}$ & $\begin{array}{c}\text { Color } \\
\text { Alone }\end{array}$ & $\begin{array}{c}\text { Dual } \\
\text { Color }\end{array}$ & $\begin{array}{c}\text { Predicted } \\
\left(\rho_{A B}=1\right)\end{array}$ \\
\hline 33 & 0.55 & 0.94 & 1.11 \\
83 & 1.09 & 1.50 & 2.17 \\
\hline
\end{tabular}

tained two elements with the form target feature value for both an SOA of $33 \mathrm{msec}[t(89)=-10.32, p<.001]$ and an SOA of $83 \mathrm{msec}[t(89)=-19.56, p<.001]$.

When the trial matrix was presented for $33 \mathrm{msec}$, there was no significant difference between $d_{\text {disparate }}^{\prime}$ and predicted independence. However, for an SOA of $33 \mathrm{msec}$, the subjects were significantly more sensitive to the coincident trials than to both the disparate trials $[t(89)=2.29$, $p=.024]$ and predicted independence $[t(89)=3.12$, $p=.002]$.

When the trial matrix was presented for $83 \mathrm{msec}$, the subjects were significantly less sensitive to disparate trials than predicted by independence $[t(89)=-3.13, p=$ $.002]$. Similarly, for an SOA of $83 \mathrm{msec}$, the subjects were significantly more sensitive to the coincident trials than to both the disparate trials $[t(89)=6.46, p<.001]$ and predicted independence $[t(89)=4.60, p<.001]$.

\section{Discussion}

The results indicate that perceptual independence between color and form fails when a trial matrix is presented for an SOA of 33 or $83 \mathrm{msec}$. At $33 \mathrm{msec}$, the subjects were significantly more sensitive to the coincident trials than to either the disparate trials or predicted independence. At $83 \mathrm{msec}$, the subjects were less sensitive to the disparate trials and more sensitive to the coincident trials than predicted by independence. Together, these results confirm the conclusion of Experiment 1 that color and form are not perceptually independent at the very early stages of perceptual analysis.

At 33 and $83 \mathrm{msec}$, two spatially disparate color targets and two spatially disparate form targets produced a weaker signal than predicted. This result supports the assumption that, although focused attention is not needed to detect the targets, relative spatial location influences target detection both within and between dimensions. This conclusion is consistent with other published reports (Moore \& Osman, 1993).

Although the results of Experiments 1 and 2 demonstrate the failure of perceptual independence, they are not perfectly parallel. In Experiment 1, perceptual independence failed only for the coincident target at $83 \mathrm{msec}$, whereas, in Experiment 2, perceptual independence failed at $33 \mathrm{msec}$ for the coincident targets and at $83 \mathrm{msec}$ for both the coincident and the disparate targets. It is important to note that, in both experiments, perceptual independence failed in an orderly manner. That is, when perceptual independence failed, the subjects detected the coincident trials better than predicted independence, and they detected the disparate trials worse than predicted in- dependence. Furthermore, both experiments reveal a positive correlation between SOA and nonindependence. The shift of the failure of perceptual independence to the shorter SOAs in Experiment 2 was probably the result of (1) the increased number of subjects and trials, which resulted in an increased sensitivity and stability, and/or (2) the higher average $d$ 's found in Experiment 2. If the higher $d$ 's influence perceptual independence, then the degree of perceptual correlation between dimensions may not be strictly a function of time. Alternatively, perceptual independence may fail at all presentation times, but the effect size for the smaller $d^{\prime}$ 's may have been too small to be detected. The implication of these results will be discussed in the General Discussion section.

\section{GENERAL DISCUSSION}

The visual detection task was designed to be a direct test of perceptual independence. In Experiments 1 and 2 , I tested whether color and form are perceptually independent at very short presentation times. The results of Experiments 1 and 2 indicate that, for processing times at least as short as $33 \mathrm{msec}$, color and form are perceptually correlated. ${ }^{1}$ This perceptual correlation manifested itself in two uniquely different processing interactions: (1) coincident targets were detected better than predicted by independence (synergy), and (2) disparate targets were detected worse than predicted by independence (inhibition). At 33 and $83 \mathrm{msec}$, there is evidence for synergy when the boundaries formed by the form and color feature dimensions are spatially coincident. At $83 \mathrm{msec}$, there is evidence for inhibition when the boundaries formed by the form and color feature dimensions are spatially disparate. Finally, inhibition is also found at both 33 and $83 \mathrm{msec}$ when two of the same target feature values are in spatially disparate locations. These data are consistent with other published research that examines the effects of redundancy and interference (Callaghan, 1984, 1989; Callaghan et al., 1986; Eriksen et al., 1989; Grice et al., 1984; Kubovy \& D. Cohen, 1991, 1992; Miller, 1982, 1991; Mordkoff \& Yantis, 1991, 1993; Mordkoff et al., 1990; Pashler, 1988; van der Heijden et al., 1984). The implications of perceptual correlation are discussed below.

\section{Implications of Perceptual Correlation}

Ashby and Townsend's (1986) general recognition theory proposes that a subject's response is a combination of the subject's perceptual and decisional processes. The perceptual processes are represented in Figure 1 by the angle between dimensions and the variance of the distri-

Table 3

Median Predicted Performance and Median $d^{\prime}$ Scores for the Form Positive Trial Matrices

\begin{tabular}{|c|c|c|c|}
\hline $\begin{array}{l}\text { Presentation } \\
\text { Time (msec) }\end{array}$ & $\begin{array}{l}\text { Form } \\
\text { Alone }\end{array}$ & $\begin{array}{l}\text { Dual } \\
\text { Form }\end{array}$ & $\begin{array}{l}\text { Predicted } \\
\left(\rho_{A B}=1\right)\end{array}$ \\
\hline 33 & 0.71 & 1.08 & 1.42 \\
\hline 83 & 1.44 & 1.88 & 2.87 \\
\hline
\end{tabular}


Table 4

Median Predicted Independence and Median $d^{\prime}$ Scores for the Dual Form and Color Positive Trial Matrices

\begin{tabular}{cccc}
\hline Presentation & & $\begin{array}{c}\text { Predicted } \\
\left(\rho_{A B}=0\right)\end{array}$ & Coincident \\
Time $(\mathrm{msec})$ & Disparate & 1.29 & 1.40 \\
\hline 33 & 1.33 & 2.07 & 2.23 \\
\hline
\end{tabular}

butions. The decisional processes are represented by the criterion the subject uses to make his or her judgment. Decisional independence holds if the criterion is parallel to the dimensional axis. The dimensional orthogonality test assesses the magnitude of $\theta$ in the perceptual space of the subjects. Therefore, if perceptual independence fails in the dimensional orthogonality test, one may conclude that its failure was the result of perceptual processes. This assertion is verified by Ashby and Townsend (1986), who make the point that "Perceptual independence is, by definition, a perceptual phenomenon, and we shall therefore wish to define it as an attribute to the perceptual system" (p.155).

Since the perceptual correlation between color and form can be attributed to perceptual processes, the next step is to determine how the perceptual system implements the perceptual correlation. It may be easier to first describe how a system would implement perceptual independence. Perceptual independence can be implemented by proposing that separate perceptual processes encode and decipher each dimension. If these processes do not interact during processing, then perceptual independence would result. For example, suppose an experiment was designed in which 2 subjects (A and B), in two separate rooms, were presented the matrices used in Experiment 1. Furthermore, suppose that only the color information was presented to Subject A and only the form information was presented to Subject B. In the signal detection model of perceptual independence, dimensional orthogonality holds if the subjects share information about the signal strength of their respective perceptions. That is, Subject A's perceived signal strength is added to Subject $B$ 's perceived signal strength, and if the combination is greater than criterion, then a target present response is made; otherwise, a target absence response is made. This is the optimal decision strategy and equals that predicted by perceptual independence (from Equation 1.1b). Independent processing systems cannot implement a decision strategy that would result in better performance. ${ }^{2}$ For performance to increase above that predicted by independence, the processing systems must interact in a more fundamental way. That is, the processing systems must share the task of processing each dimension, at least to some degree. Thus, perceptual correlation indicates interaction between the dimensional processing systems.

To understand how perceptual correlation may be implemented, imagine a situation in which the processing systems were perfectly correlated. In such a situation, each system would process complete information from both dimensions. Using the previous example, both Subject $A$ and Subject B would be shown complete color and complete form information. Because, in this example, each subject represents a single processing system, we assume that color and form are processed together in that system, and, therefore, color and form information are not differentiated within the system. In this instance, the signal strength for a single-target trial would increase slightly, but the signal strength for a dual-target trial would have a greater proportional increase. For example, suppose that Subject A's sensitivity to the color-alone target and the form-alone target was $d_{C}^{\prime}=1.0$, and $d_{F}^{\prime}=1.0$, respectively. Furthermore, suppose that Subject B's sensitivity to those targets was the same. Then, the pair's sensitivity to the each single-target trial type would be 1.41 (from Equation 1.1b). Because each processing system (Subjects A and B) processes both color and form information together, each processing system's sensitivity to the dual-target trials would be $d_{C F}^{\prime}=2.0$. (Because color and form information are not differentiated within each system, a trial with two targets present would equal $d_{C}^{\prime}{ }^{+}$ $d_{F}^{\prime}$.) The independent combination of the pair's sensitivity to the dual-target trials would be 2.82 . This is exactly double the pair's sensitivity to the single-target trials. This result is predicted by Equation 1.1 a for perfectly correlated processing systems $\left(\rho_{A B}=1, \theta=0^{\circ}\right)$. Thus, perfectly correlated processing systems indicate that each system processes complete information for both dimensions. Such a system is theoretically equivalent to a single system that processes both dimensions. In such a system, inhibition would be implemented by a negative correlation between dimensions. That is, increasing the signal strength of dimension $A$ would actively decrease the signal strength of dimension $B$.

The perceptual correlation between color and form found in Experiments 1 and 2 was greater than perceptual independence but was less than a perfect correlation. This indicates that there are two processing systems, and each system specializes in the processing of a specific dimension (one for color and one for form). However, the results also indicate that each system has access to, and processes, some information from the dimension that it does not specialize in. Thus, if we refer back to our two processing systems in the previous example (Subject A and Subject B), Subject A would be shown all of the color information plus some of the form information, whereas Subject B would be shown all of the form information plus some of the color information. In this instance, the signal strength in a single-target trial would increase slightly, but the signal strength in the dual-target trials would increase beyond that predicted by independence (but less than that predicted by perfect correlation).

In summary, if one accepts the signal detection model of perceptual space, then perceptual correlation between two dimensions cannot be predicted by independent processing systems. The systems processing each dimension must process some information about the other dimension for sensitivity to a dual-target trial to increase beyond that predicted by independence. Therefore, perceptual correlation indicates interaction between processing systems. This interaction can be direct, or it may be the 
result of a third variable. If a third variable is involved, the third variable must interact with each processing system during the processing of the dimensions (e.g., not after processing is complete). Even though the processing systems remain technically autonomous when a third variable is involved, they are not independent, because information is shared between the systems (albeit through a third variable) during perceptual processing. Thus, the addition of a third variable does not change the conclusion that the processing systems interact during processing. Evidence for a neurological foundation for this interaction is presented below.

\section{Neuroanatomy}

The data indicate that the color and form processing systems interact in a fundamental way. That is, some form information is processed in the color system, and some color information is processed in the form system. Although there is some evidence that the neuroanatomical makeup of the visual system supports independent processing systems for different dimensions (e.g., Livingstone \& Hubel, 1984, 1987, 1988), there are extensive data indicating that independent processing systems are unlikely (e.g., Felleman \& Van Essen, 1991; Zeki \& Shipp, 1988). The claims for independent processing systems comes from the neuroanatomical data, which show that neurons that are maximally sensitive to specific feature dimensions form pathways that begin at the retina and continue throughout most of the visual system (for reviews, see Felleman \& Van Essen, 1991; Livingstone \& Hubel, 1988; Zeki \& Shipp, 1988). These pathways are called processing streams. The visual system has three robust processing streams: the magnocellular stream, the parvo-blob stream, and the parvo-interblob stream. The neurons in the magnocellular stream are highly sensitive to brightness contrast and have fast response times, but they discriminate detail and wavelength poorly. These cells are maximally sensitive to moving stimuli. The neurons in the parvo-blob stream are highly sensitive to wavelength, have slower response times, and a reduced sensitivity to brightness contrast. These cells are maximally sensitive to color. The neurons in the parvo-interblob stream are highly sensitive to detail, have slower response times, and a reduced sensitivity to brightness contrast. These cells are maximally sensitive to line orientation. The continued segregation of these processing streams over many hierarchical cortical areas has been interpreted as evidence that feature dimensions are processed independently (Hubel \& Livingstone, 1990; Livingstone \& Hubel, 1984, 1987, 1988).

However, there is extensive data that demonstrate a high degree of interconnectivity between the visual streams and visual areas (DeYoe \& Van Essen, 1988; Felleman \& Van Essen, 1991; Merigan \& Maunsell, 1993; Schiller, Logothetis, \& Charles, 1991; Van Essen, Anderson, \& Felleman, 1992; Zeki, 1992; Zeki \& Shipp, 1988). There are 32 distinct neocortical areas involved in the processing of visual information. If these areas were fully networked (each area connected with every other area), then a total of $992[N(N-1)]$ pathways would exist (Felleman \& Van Essen, 1991). Between 30\% and $45 \%$ of those pathways have already been identified (Felleman \& Van Essen, 1991). A majority of these pathways are bidirectional, indicating reciprocally interconnected areas. Extensive cross-connections between pathways are found as early as area V1, and low level visual information (carried by the processing streams) appears to be almost fully combined in the cortical temporal pathway (see Merigan \& Maunsell, 1993). These numerous interstream connections provide the neurological foundation for stream interaction.

\section{Psychophysical Models of the Early Visual System}

Many psychophysical models of early vision make either implicit or explicit assumptions about whether perceptual independence holds for color and form. As noted above, perceptual correlation between dimensions can result only from an interaction between the systems that process each dimension. Therefore, models of perception that claim processing independence of feature dimensions assume perceptual independence between processing systems. In addition, the differential detection rates produced by the two dual-target trial types demonstrate that the interaction between the form and color processing systems (at least at presentation times of $33 \mathrm{msec}$ or greater) includes information about relative spatial location of each target dimension. This interaction was manifested by the synergistic reaction when the dimensions produce boundaries in spatially coincident locations, and the inhibitory reaction when the dimensions produce boundaries in spatially disparate locations. Therefore, to be consistent with the present data, models of early vision must also implement a system that takes into account the relative spatial location of color and form information.

Stage theories are probably the most well known theories of perception that discuss dimensional processing. Most stage theories of perception propose that perceptual analysis begins with an unlimited-capacity stage, often termed early vision, preattention, or diffuse attention, and is followed by a limited-capacity stage, often termed $f o$ cused attention or spatial attention (Cave \& Wolfe, 1990; Duncan, 1980; Monheit \& Johnson, 1994; Neisser, 1966; Treisman \& Gelade, 1980; Treisman \& Sato, 1990; Wolfe, Cave, \& Franzel, 1989). What differentiates these theories are the nature of perceptual processing that occurs in each of these stages and when information is available for identification. This debate has centered on the issue of early versus late selection. The selection issue examines what type of information can be selected to pass from the unlimited-capacity stage to the limitedcapacity stage.

Early selection. Probably the most well known early selection theories are feature integration theory (FIT; Treisman \& Sato, 1990) and Guided Search 2 (GS2; Wolfe, 1994). The early selection account holds that only simple stimulus characteristics, such as feature dimensions, are processed during early vision (e.g., Treisman \& Sato, 1990; Wolfe, 1994). Therefore, only individual feature 
dimensions can be used to select stimuli to be passed on to the limited-capacity stage. The limited-capacity stage combines the feature dimensions into objects and, in doing so, completes the analysis of the stimuli.

Although often not explicitly stated, this account implies processing independence between feature dimensions during early vision (if feature dimensions were functionally dependent, then selection based on that dependence should be possible). This point is made explicit when Treisman (1993) defines features as

properties for which we have evolved or acquired separate sets of detectors responding in parallel across the visual scene. The detectors need not be individual neurons; they could be groups or hierarchies of cells so long as they respond directly to their particular properties without attentional control and without (much) crosstalk from other functional detectors. (p. 7, italics added)

Wolfe (1994) also makes this point explicit in his description of the computer model of GS2.

The results of Experiments 1 and 2 contradict the claim of processing independence between color and form, at least for presentation times of $33 \mathrm{msec}$ or more. FIT and GS2 currently have no mechanism for the interaction between processing systems during dimensional processing and, therefore, do not predict the synergistic and inhibitory effects that are based on the spatial relationship between targets. GS2 does, however, have a mechanism for combining the signal strength output of each processing system. This combination is completed in the activation map (Wolfe, 1994). The activation map is retinotopic and contains a weighted sum of the activations from the independent processing systems (termed feature maps). The implementation of the activation map is exactly the optimal decision strategy for independent processing systems described above. As discussed above, such a system cannot produce the synergistic effect found for the coincident conditions. That is, such a system cannot perform at better than predicted independence.

In sum, these and other theories that assume independent dimensional processing systems cannot be implemented so as to produce perceptual correlation between dimensions. It is unclear whether a modification of these theories to include the interaction between dimensional processing systems would undermine any assumptions of the early selection account. Indeed, such a modification may be premature given the evidence only from the present experiments. However, as discussed in the introduction, the present experiments are just a few of the numerous recent experiments that provide evidence against independent processing systems.

Late selection. The late selection account holds that stimuli are fully identified and characterized during early vision (e.g., Allport, Tipper, \& Chmiel, 1985; Duncan, 1980). Therefore, any aspect of the stimulus can be used to select it to be passed on to the limited-capacity stage. The limited-capacity stage is necessary, not for further processing of the stimuli but to bring the presence of the stimuli into awareness. The late selection ac- count makes no explicit assumption about the degree of perceptual independence during early vision. However, complete processing of stimuli during early vision implies that all feature dimensions are fully integrated preattentivly (see Allport et al., 1985). The late selection account, as initially stated (e.g., Duncan's, 1980, combined condition), would predict independent detection of the two targets, since each target enters the limited-capacity stage independently. However, if all stimuli are fully integrated during the early stage (e.g., Allport et al., 1985), then there is no reason to assume that the targets are passed to the limited-capacity stage independently. Without this assumption, theories based on the late selection account can accommodate the present data.

It appears that the late selection account can most easily explain the data. Unfortunately, while most perceptual theories based on early selection are fairly detailed in describing the processing that occurs in the early stage of perceptual analysis (e.g., FIT and GS2), those based on the late selection account are vague in this respect. In addition, the early selection accounts can be modified so as not to predict processing independence. Below, I present a theory of active signal modulation that may explain the data. It should be noted that the theory presented below is just one implementation of numerous theories that may explain the data.

\section{A Model of Active Signal Modulation}

The differential detection rates produced by the two dual-target trial types demonstrate that the interaction between the form and color processing systems includes information about relative spatial location of each target dimension. This interaction manifests itself by the synergistic reaction when the dimensions produce boundaries in coincident locations, and the inhibitory reaction when the dimensions produce boundaries in spatially disparate locations. This data indicates that the algorithm used by the visual system to sum signals within and between dimensions actively modulates the signal strength of the sense data. In the coincident condition, the two stimuli could not alone produce a signal as strong as the detection rates indicated. This suggests that signal strength is amplified within the system. In addition, the visual system actively attenuates the signal of elements in different spatial locations. This attenuation is not debilitating since the combined signals are still detected better than the strongest individual signal. The attenuation of signal strength was revealed both when summing signals across feature dimensions and when summing signals within feature dimensions. The consistency of the summing algorithm within and between dimensions may suggest a single networked system. To posit multiple systems with identical tasks is computationally expensive.

By actively modulating the signal strength of the sense data, the visual system is distorting the environment. In terms of selective adaptation, active distortion is risky business. The distortion should enhance only what is real and attenuate only noise. The modulation should therefore be based on the visual structure of the environment. 
Objects in the environment tend to generate coincident boundaries in different feature dimensions. That is, feature values of form, color, texture, motion, and so on, all produce spatially similar boundaries around single objects. A visual system that internalizes this assumption may have evolutionary advantages, because an increased sensitivity to objects would translate into an increased sensitivity to the environment. Such a system would show the modulation function described by the data of Experiments 1 and 2 (i.e., the amplification of coincident boundaries and inhibition of disparate boundaries).

Modulation of the sense data by the visual system is not uncommon. The visual system modulates contrast boundaries, spatial frequency, color, and so on. However, most modulation occurs in or near the retina. The present data reveal a modulation transfer function that may take a higher level input (i.e., boundaries) and modulate the signal of an abstract concept (i.e., "objectness"). The specific mechanism by which this modulation takes place is unknown. However, the data seem to indicate that each processing stream begins to process the relevant sense data. As discontinuities between different feature values of the same feature dimension (boundaries) begin to form, the location of these boundaries is shared with other streams. The consistency of the boundary information received from other processing streams may be used as a basis for the modulation of the signal strength of the sense data. Sense data that indicate boundaries in spatially coincident locations would be amplified, and sense data that indicate boundaries in spatially disparate locations would be attenuated. This would result in the amplification of "objects" and the attenuation of noise. The gradual relaxation toward a solution would explain the positive correlation between SOA and perceptual correlation.

This active signal modulation model of early vision may be a parsimonious explanation of both present and past results. There is a neuroanatomical foundation for stream interaction. The elimination of a theoretical second stage that combines dimensions in favor of direct interstream interaction provides a solution for the empirically demonstrated time constraints (perceptual correlation at times at least as small as $33 \mathrm{msec}$ ). A network in which processing streams share information about boundary location and strength would explain the inhibitory and synergistic pattern of interaction revealed in Experiments 1 and 2. This pattern of interaction is predictable from a description of the organization of the visual world. Thus, the network architecture effectively accounts for the data presented and elegantly mirrors the visual environment.

\section{Future Directions}

There are, of course, limitations to the present study. First, the colors used were not isoluminant. This is common in the psychophysical literature addressing this issue (e.g., Duncan, 1980, 1985; Eriksen et al., 1989; Grice et al., 1984; Miller, 1982, 1991; Monheit \& Johnson, 1994; Mordkoff \& Yantis, 1991, 1993; Mordkoff et al., 1990; Treisman, 1985; Treisman \& Gelade, 1980; Treisman \& Gormican, 1988; van der Heijden et al., 1984;
Wolfe, 1994; Wolfe et al., 1989). Indeed, most of the data that the present theories are based on are a function of colors that are not isoluminant. Nevertheless, it is important to replicate the present findings using isoluminant stimuli. I am currently conducting experiments that address this issue. A further limitation is that only two presentation times were used ( 33 and $83 \mathrm{msec}$ ). Although perceptual independence failed at $33 \mathrm{msec}$ in Experiment 2, it is possible that perceptual independence would hold at shorter times. Again, experiments are under way to address this issue.

\section{CONCLUSION}

In summary, Experiments 1 and 2 provide direct evidence that the form and color feature dimensions are not processed independently at times greater than $33 \mathrm{msec}$. The data revealed a pattern of synergy and inhibition between feature dimensions that was linked to their relative spatial location. This pattern of results constrains all models of early vision. A model of early vision based on active signal modulation was proposed.

\section{REFERENCES}

Allport, D. A., Tipper, S. P., \& Chmiel, N. R. J. (1985). Perceptual integration and postcategorical filtering. In M. I. Posner \& O. S. M. Marin (Eds.), Attention and performance XI (pp. 107-132). Hillsdale, NJ: Erlbaum.

ASHBy, F. G., \& ToWnSEND, J. T. (1986). Varieties of perceptual independence. Psychological Review, 93, 154-179.

Butler, B. E., MEWhORT, D. J. K., \& BrowSE, R. A. (1991).When do letter features migrate? A boundary condition for feature-integration theory. Perception \& Psychophysics, 49, 91-99.

Callaghan, T. C. (1984). Dimensional interaction of hue and brightness in preattentive field segregation. Perception \& Psychophysics, 36, 25-34.

Callaghan, T. C. (1989). Interference and dominance in texture segregation: Hue, geometric form, and line orientation. Perception \& Psychophysics, 46, 299-311.

Callaghan, T. C., Lasaga, M. I., \& Garner, W. R. (1986). Visual texture segregation based on orientation and hue. Perception \& Psychophysics, 39, 32-38.

CAVE, K. R., \& Wolfe, J. M. (1990). Modeling the role of parallel processing in visual search. Cognitive Psychology, 22, 225-271.

COHEN, A., \& IVRY, R. (1989). Illusory conjunctions inside and outside the focus of attention. Journal of Experimental Psychology: Human Perception \& Performance, 15, 650-663.

COHEN, J. (1994). The earth is round $(p<.05)$. American Psychologist, 49, 997-1003.

De YoE, E. A., \& VAN EssEn, D. C. (1988). Concurrent processing streams in monkey visual cortex. Trends in Neurosciences, 11, 219-226.

Duncan, J. (1980). The locus of interference and the perception of simultaneous stimuli. Psychological Review, 87, 271-300.

Duncan, J. (1985). Visual search and visual attention. In M. I. Posner \& O. S. M. Marin (Eds.), Attention and performance XI (pp. 85-106). Hillsdale, NJ: Erlbaum.

ERIKSEN, C. W. (1988). A source of error in attempts to distinguish coactivation from separate activation in the perception of redundant targets. Perception \& Psychophysics, 44, 191-193.

Eriksen, C. W., Goettl, B., St. James, J. D., \& Fournier, L. R. (1989). Processing redundant signals: Coactivation, divided attention, or what? Perception \& Psychophysics, 45, 356-370.

Felleman, D. J., \& Van Essen, D. C. (1991). Distributed hierarchical processing in the primate cerebral cortex. Cerebral Cortex, 1, 1-47.

Green, D. M., \& Swets, J. A. (1966). Signal detection theory and psychophysics. New York: John Wiley. 
Grice, G. R., Canham, L., \& Boroughs, J. M. (1984). Combination rule for redundant information in reaction time tasks with divided attention. Perception \& Psychophysics, 35, 451-463.

HubEL, D. H., \& Livingston, M. S. (1990). Color contrast sensitivity in the lateral geniculate body and primary cortex of the macaque monkey. Journal of Neuroscience, 10, 2223-2237.

ISENBERG, L., \& NisSEN, M. J., \& MARCHAK, L. C. (1990). Attentional processing and the independence of color and orientation. Journal of Experimental Psychology: Human Perception \& Performance, 16, 869-878.

IVRY, R. B., \& Prinzmetal, W. (1991). Effect of feature similarity on illusory conjunctions. Perception \& Psychophysics, 49, 105-116.

KadLEC, H., \& Townsend, J. T. (1992). Signal detection analysis of dimensional interactions. In F. G. Ashby (Ed.), Multidimensional models of perception and cognition (pp. 181-227). Hillsdale, NJ: Erlbaum.

Kubovy, M., \& CohEN, D. [J.] (1991, November). Are texture segregation by form and color independent of each other? Paper presented at the 32nd Annual Meeting of the Psychonomic Society, San Francisco.

Kubovy, M., \& CohEN, D. [J.] (1992, November). Further tests of feature-module architectures for color and form. Paper presented at the 33rd Annual Meeting of the Psychonomic Society, St. Louis.

Livingstone, M. S., \& Hubel, D. H. (1984). Anatomy and physiology of the color system in the primate visual cortex. Journal of Neuroscience, 4, 309-356.

Livingstone, M. S., \& Hubel, D. H. (1987). Connections between layer $4 \mathrm{~B}$ of area 17 and the thick cytochrome oxidase stripes of area 18 in the squirrel monkey. Journal of Neuroscience, 7, 3371-3377.

Livingstone, M. [S.], \& Hubel, D. [H.] (1988). Segregation of form, color, movement, and depth: Anatomy, physiology, and perception. Science, 240, 740-479.

Macmillan, N. A., \& Creelman, C. D. (1991). Detection theory: A user's guide. Cambridge: Cambridge University Press.

Merigan, W. H., \& Maunsell, J. H. R. (1993). How parallel are the primate visual pathways? Annual Review of Neuroscience, 16, 369.402.

Miller, J. (1982). Divided attention: Evidence for coactivation with redundant signals. Cognitive Psychology, 14, 247-279.

MiLLeR, J. (1991). Channel interaction and the redundant-target effect in bimodal divided attention. Journal of Experimental Psychology: Human Perception \& Performance, 17, 160-169.

Monheit, M. A., \& Johnson, J. C. (1994). Spatial attention to arrays of multidimensional objects. Journal of Experimental Psychology: Human Perception \& Performance, 20, 691-708.

Moore, C. M., \& Osman, A. M. (1993). Looking for two targets at the same time: One search or two? Perception \& Psychophysics, 53, $381-390$

MoRdKofF, J. T., \& YANTIS, S. (1991). An interactive race model of divided attention. Journal of Experimental Psychology: Human Perception \& Performance, 17, 520-538.

MordKoff, J. T., \& YANTIS, S. (1993). Dividing attention between color and shape: Evidence of coactivation. Perception \& Psychophysics, 53, 357-366.

Mordkoff, J. T., Yantis, S., \& Egeth, H. E. (1990). Detecting conjunctions of color and form in parallel. Perception \& Psychophysics, 48, 157-168.

Neisser, U. (1966). Cognitive psychology. New York: Appleton-CenturyCrofts.

NISSEN, M. J. (1985). Accessing features and objects: Is location special? In M. I. Posner \& O. S. M. Marin (Eds.), Attention and performance $X I$ (pp. 205-219). Hillsdale, NJ: Erlbaum.

PASHLER, H. (1988). Cross-dimensional interaction and texture segregation. Perception \& Psychophysics, 43, 307-318.
Rosenthal, R., \& RuBIN, D. B. (1986). Meta-analytic procedures for combining studies with multiple effect sizes. Psychological Bulletin, 99, 400-406.

Schiller, P. H., Logothetis, N. K., \& Charles, E. R. (1991). Parallel pathways in the visual system: Their role in perception at isoluminance. Neuropsychologia, 29, 433-441.

SCHWARZ, W. (1989). A new model to explain the redundant-signals effect. Perception \& Psychophysics, 46, 498-500.

Strube, M. J. (1985). Combining and comparing significance levels from nonindependent hypothesis tests. Psychological Bulletin, 97, 334-341.

Strube, M. J., \& Miller, R. H. (1986). Comparison of power rates for combined probability procedures: A simulation study. Psychological Bulletin, 99, 407-415.

Treisman, A. (1985). Preattentive processing in vision. Computer Vision, Graphics, \& Image Processing, 31, 156-177.

Treisman, A. (1993). The perception of features and objects. In A. D. Baddeley \& L. Weiskrantz (Eds.), Attention: Selection, awareness, and control. A tribute to Donald Broadbent (pp. 5-35). Oxford: Oxford University Press.

Treisman, A., \& Gelade, G. (1980). A feature integration theory of attention. Cognitive Psychology, 12, 97-136.

Treisman, A., \& Gormican, S. (1988). Feature analysis in early vision: Evidence from search asymmetries. Psychological Review, 95, 15-48.

Treisman, A., \& SATo, S. (1990). Conjunction search revisited. Journal of Experimental Psychology: Human Perception \& Performance, 114, 285-310.

Treisman, A., \& SchmidT, H. (1982). Illusory conjunctions in the perception of objects. Cognitive Psychology, 14, 107-141.

van der Heijden, A. H. C., Maris, S. L., \& NeerincX, M. (1984). Some evidence for correlated separate activation in a simple letterdetection task. Perception \& Psychophysics, 36, 577-585.

Van Essen, D. C, Anderson, C. H., \& Felleman, D. J. (1992, January). Information processing in the primate visual system: An integrated systems perspective. Science, 255, 419-423.

WOLFE, J. M. (1994), Guided Search 2.0: A revised model of visual search. Psychonomic Bulletin \& Review, 1, 202-238.

Wolfe, J. M., CAve, K. R., \& Franzel, S. L. (1989). Guided search: An alternative to the feature integration model of visual search. Journal of Experimental Psychology: Human Perception \& Performance, $15,419-433$.

ZEKI, S. (1992). The visual image in mind and brain. Scientific American, 267, 69-76.

ZEKI, S., \& SHIPP, S. (1988). The functional logic of cortical connections. Nature, 335, 311-317.

\section{NOTES}

1. It should be noted that there is always a chance that the dimensions were perceptually independent at an earlier point in time. Therefore, although the results show perceptual correlation between the color and form feature dimensions during the initial unlimited-capacity stage of vision, perceptual independence may be found at presentation times less than $33 \mathrm{msec}$.

2. Aside from being mathematically determined to be the optimal strategy, I also ran a computer simulation that confirmed this.

(Manuscript received March 13, 1995; revision accepted for publication May 31, 1996.) 